$12-2020$

\title{
Innovating and validating measures of complex concepts
}

Evidence Project

Follow this and additional works at: https://knowledgecommons.popcouncil.org/departments_sbsr-rh How does access to this work benefit you? Let us know!

\section{Recommended Citation}

Evidence Project. 2020. "Innovating and validating measures of complex concepts," Learnings from the Evidence Project. Washington, DC: Population Council, The Evidence Project.

This Brief is brought to you for free and open access by the Population Council. 


\section{BOX 1: DOMAINS OF QOC}

1. Respectful care: interpersonal relations between provider and client; treating client with dignity and respect; ensuring audiovisual privacy and confidentiality

2. Method selection: solicitation of information from client about reproductive intentions, previous contraceptive use, and other domains; provision of information by provider on various FP methods

3. Effective use: information given about how to use the method, potential side effects, how to manage side effects, and warning signs

4. Continuity of contraceptive use and care: information given on whether to return for follow-up, other sources of FP supply, and possibility of switching methods

in a longitudinal study of contraceptive use dynamics in India. Exploratory factor analysis was used to examine the psychometric properties of the items and factors. The 22 items were reduced to 10 . Both measures' reliability was assessed using Cronbach's alpha. Overall QoC scores were calculated for both the 22- and 10-items measures, and then categorized into low, medium, and high. Women who reported medium or high QoC (as measured by both the 22- and 10-item measures) at contraceptive initiation were significantly more likely to be using a modern contraceptive method three months later, compared to women who received low QoC (Figure 1).

\section{OUR IMPLEMENTATION SCIENCE APPROACH}

The Evidence Project used implementation science (IS)-the strategic generation, translation, and use of evidence-to strengthen, scale-up, and sustain voluntary family planning (FP) services.

Our IS approach was driven by collaboration with partners to: (1) define research priorities; (2) implement research activities; and (3) use evidence for decision-making.

This brief is part of a series of briefs synthesizing evidence and lessons learned through the project. Other briefs include:

- Expanding access and method choice through private sector provision and self-administered injectables

- Evidence for expanding access to voluntary FP through workplace interventions

These briefs and all other Evidence Project publications can be found at: http://evidenceproject.popcouncil.org/.

The 10-item version adequately measured QoC, making it ideal for routine data collection and monitoring of programs. In Burkina Faso and Mali, the Evidence Project is exploring how to use these measures to routinely monitor QoC for FP services in the public sector and through performance-based financing programs. The 22-item measure is a more comprehensive way to measure QoC, and is best suited for more specialized studies.

FIGURE 1. MODERN METHOD CONTINUATION 3 MONTHS LATER BY QUALITY COMPOSITE SCORE AMONG WOMEN AGED 15-49 IN INDIA $(\mathrm{N}=2,306)$

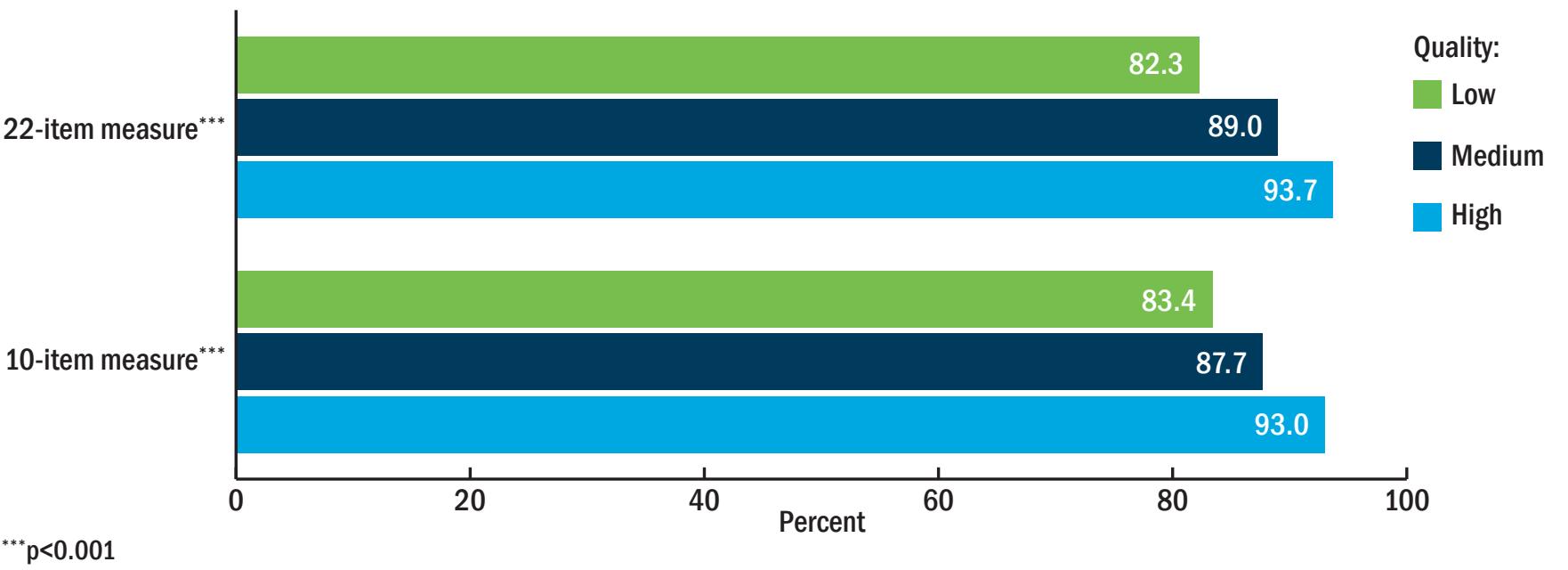




\section{Method Information Index Plus}

The Method Information Index (MII) is a measure designed to capture informed choice at FP method initiation and is a core indicator to monitor progress toward national and international FP goals. The MII is operationalized as the percentage of current FP users who report being told about 1) other methods, 2) the side effects of the method selected, and 3) how to manage side effects at the time they adopted their current method. Using the same longitudinal study in India, the Evidence Project assessed the value of adding a fourth question to assess whether the FP client was told about the possibility of switching to a different contraceptive method if the current method was no longer suitable. With the addition of this fourth measure, the MII was more predictive of contraceptive continuation than the three-item MII. The MIIplus (four-item measure) has been added to the Demographic and Health Surveys and to Performance Monitoring for Action Surveys to routinely measure informed choice across countries and over time.

More information on the QoC measures is available in "Metrics for monitoring and improving quality of care in family planning" brief.

\section{Anticipated stigma}

The Evidence Project conducted a study among married 15- to 24-year-old females in Ethiopia with a demand (met and unmet need) for FP to better understand how

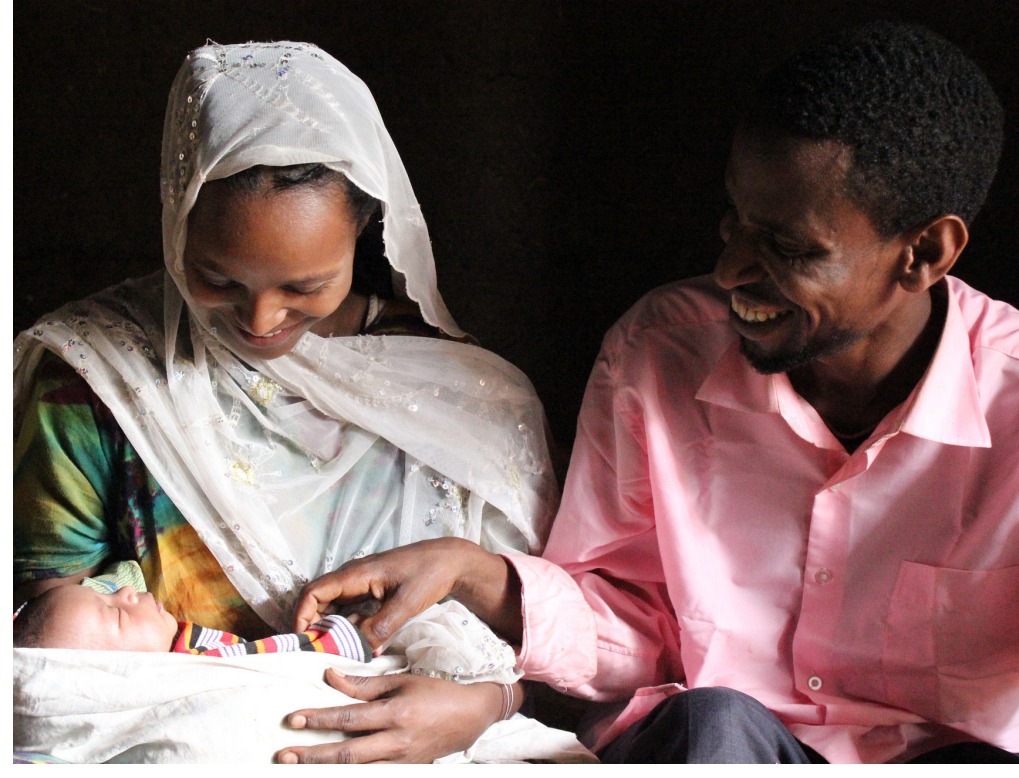

anticipated stigma influences voluntary FP use. The study included five items of anticipated stigma. Results of the study found that overall, 55 percent of respondents agreed with at least one item of the anticipated stigma scale. Furthermore, youth who answered positively on at least one item of anticipated stigma were significantly more likely to have an unmet need for FP. The association between anticipated stigma and unmet need remained significant even after adjusting for other known barriers to FP use. While further work should be done to see whether anticipated stigma predicts met and unmet need, results from this study suggest anticipated stigma is potentially a significant barrier to satisfying demand for FP. More information on this anticipated stigma tool is available in "Measurement of anticipated stigma: A barrier to family planning use among married youth" brief.

\section{FIGURE 2. TOP SCALES USED IN DATABASE}

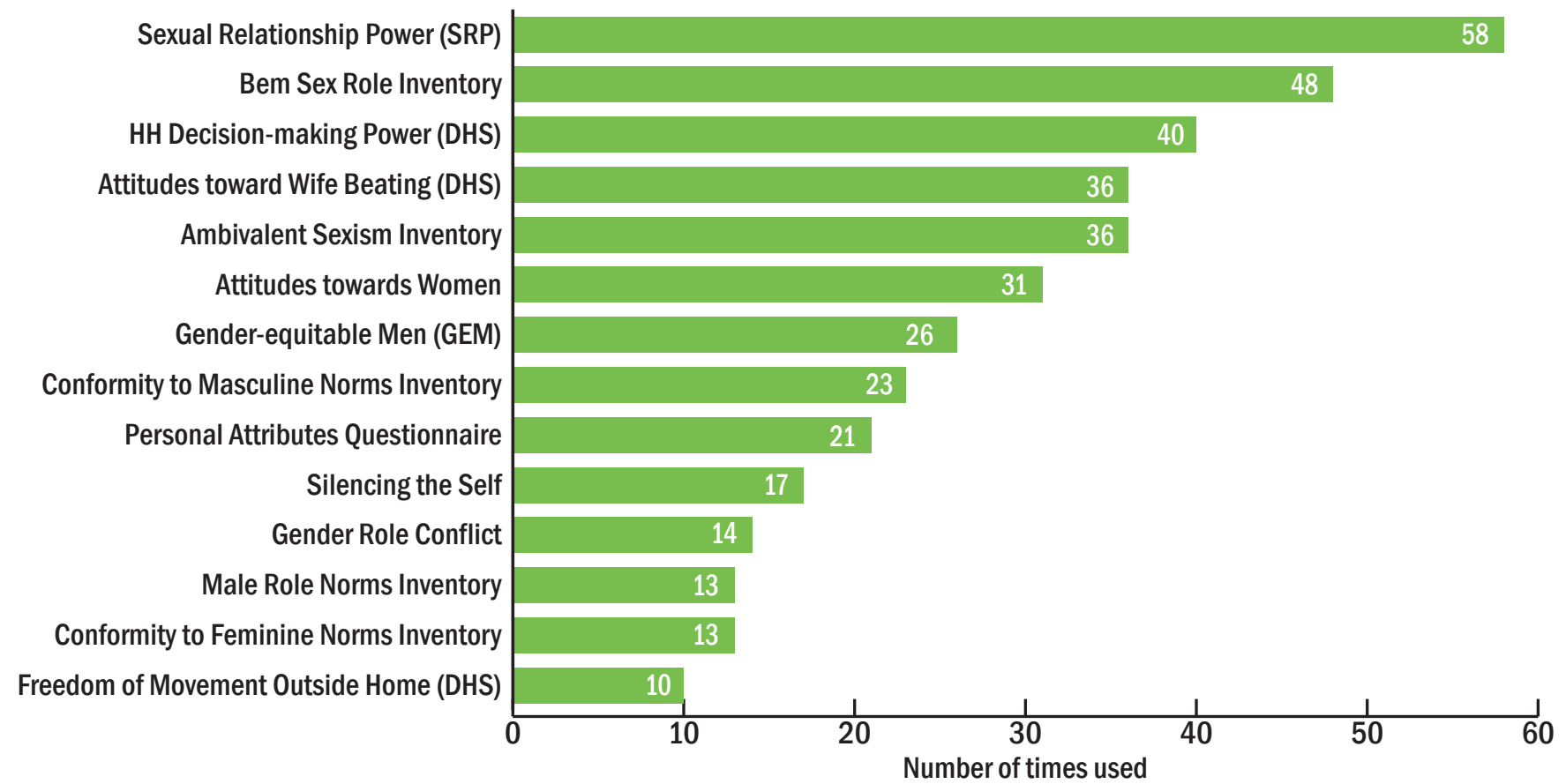




\section{Gender and power metrics}

By systematically searching five electronic databases, supplemented by hand searches of specific journals and conference paper repositories for relevant titles, the Evidence Project compiled a database of scales and measures to be used by researchers and program implementers to measure gender and power. The database now houses over 600 unique measures, many tested in multiple settings and used in many studies (Figure 2). The database includes multiitem scales and single-item questions that reflect gender norms, personal views or beliefs about gender roles and norms, gender role stress, gendered-dynamics, power and control in relationships, and individual-level agency and self-efficacy, among others. The Gender and Power Metrics database provides information on the settings and populations where gender and power measures have been implemented, references for the published articles, as well as extracted information on scale psychometric properties and, where available, the question(s) that comprise the measure. In cases where gender or power scales were measured in association with family planning or IPV outcomes, the database provides additional information regarding the quantitative association between both constructs. The database is open-access and can be explored here: https://gendermetrics.popcouncil.org/, and more information about the database is available in "Gender and Power Metrics Database: A living database of scales that have been used to measure constructs of gender, agency, power, and control" brief.

\section{Reference}

1. Jain, Anrudh K., John Townsend, and Saumya RamaRao. 2018. "Proposed metrics to measure quality: An overview," Working Paper no. 3, prepared for the Measuring and Monitoring Quality of Care Project. New York: Population Council.

\section{Acknowledgments}

This brief summarizes key findings from several studies and activities conducted by the Evidence Project/Population Council team, led by... Aparna Jain (Quality of Care and Anticipated Stigma) and Nicole Haberland (Gender Metrics) with contributions from Rajib Acharya, Kumudha Aruldas, Sara Chace Dwyer, Annabel Erulkar, Hussein Ismael, Arupendra Mozumdar, and Elizabeth Tobey. In addition, we are grateful to Michelle Hindin, Sherry Hutchinson, Leah Jarvis, and Erika Martin for their contributions to this brief. We acknowledge the generous support of USAID Washington as well as the USAID Missions of India and Ethiopia for these studies, and we are grateful to the women and young people who gave their time and shared their experiences.

\section{FURTHER RESOURCES}

\section{Quality of care}

Jain, A., K. Aruldas, A. Mozumdar, E. Tobey, \& R. Acharya. 2019. "Validation of two quality of care measures: Results from a longitudinal study of reversible contraceptive users in India," Studies in Family Planning 50(2): 179193. doi: 10.1111/sifp.12093

Jain, A., K. Aruldas, E. Tobey, A. Mozumdar, \& R. Acharya. 2019. "Adding a question about method switching to the method information index is a better predictor of contraceptive continuation," Global Health: Science and Practice 7(2): 289-299. doi: 10.9745/GHSP-D-19-00028

\section{Anticipated stigma}

Jain, A., H. Ismail, E. Tobey, \& A. Erulkar. 2019. "Stigma as a barrier to family planning use among married youth in Ethiopia," Journal of Biosocial Science 51(4): 505519. doi: 10.1017/S0021932018000305

\section{Gender and power}

McCarthy, K. J., R. Mehta, \& N. A. Haberland. 2018. "Gender, power, and violence: A systematic review of measures and their association with male perpetration of IPV," PIoS One 13(11). doi: 10.1371/journal.pone.0207091

\section{THE EVIDENCE PROJECT} Population Council 4301 Connecticut Ave. NW Washington, DC 20008 tel: +202 2379400 evidenceproject@popcouncil.org

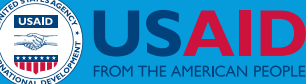

The Evidence Project is made possible by the generous support of the American people through the United States Agency for International Development (USAID) under the terms of cooperative agreement no. AID-OAA-A-13-00087. The contents of this document are the sole responsibility of the Evidence Project and Population Council and do not necessarily reflect the views of USAID or the United States Government.

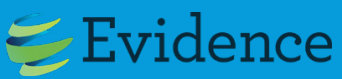

The Evidence Project uses implementation science-the strategic generation, translation, and use of evidence-to strengthen and scale up family planning and reproductive health programs to reduce unintended pregnancies worldwide. The Evidence Project is led by the Population Council.

Suggested Citation: The Evidence Project. 2020. “Innovating and validating measures of complex concepts, Learnings from the Evidence Project. Washington, DC: Population Council, The Evidence Project.

(c) 2020 The Population Council, Inc.

Photo credits: Cover-CPippa Ranger/Department for International Development (CC BY-NC-ND 2.0) Page 3-@Adey Abebe, JSI Last Ten Kilometers project (CC BY-NC-SA 2.0) 